\section{Gericht verpflichtet Ärzte zur Online-Abrechnung}

Die Online-Abrechnung bleibt Pflicht. Das hat das Landessozialgericht Niedersachsen-Bremen (LSG) entschieden (Aktenzeichen L 3 KA 25/11 B ER).

Ein Internist hatte vor dem Bremer Sozialgericht geklagt. Er wollte die Abrechnung weiter auf Diskette abliefern. Grund: Die Online-Abrechnung sei unsicher und finanziell unzumutbar. Das SGB V erlaube beide Wege der Datenübertragung. Der Arzt beantragte eine einstweilige Anordnung, die Daten auch künftig per Diskette einreichen zu dürfen. Das SG lehnte ab. Nun scheiterte der Arzt auch vor dem LSG. Christian Beneker

\section{KV-Verwaltungsakt gegen Kasse rechtens}

Eine KV darf über den Antrag einer Krankenkasse auf Honorarberichtigung mit Verwaltungsakt entscheiden. Nach Auffassung des Bundessozialgerichts (BSG) in Kassel ist dies im Interesse der Rechtssicherheit für betroffene Ärzte wichtig. Denn nach einem Verwaltungsakt bleibt genau ein Monat Zeit, Rechtsmittel einzulegen, danach wird der Akt rechtskräftig (Aktenzeichen: B 6 KA 30/10 R).

Im konkreten Fall hatte die KKH Allianz die Abrechnungen mehrerer Zahnärzte beanstandet. Den Antrag auf Honorarberichtigung lehnte die KZV Hessen jedoch überwiegend ab. Gegenüber der Kasse erließ sie einen entsprechenden Verwaltungsakt. Martin Wortmann

\section{Keine Rezepte bei ruhender Approbation}

Ruht die Approbation, dürfen Ärzte keine Rezepte ausstellen, so das Sozialgericht (SG) Düsseldorf (Aktenzeichen S 2 KA 227/11 ER). Schummelt ein Arzt, kann das zum Aus der Praxis führen.

In konkreten Fall ruhte die Approbation eines Arztes. Er führte die Praxis mit einem Vertreter fort, bis die KV die Genehmigung dafür zurückzog. Die Staatsanwaltschaft hatte festgestellt, dass der Praxisinhaber Rezepte ausgestellt hatte. Er sagte zwar, er sei nicht ärztlich tätig geworden. Das SG glaubte inm aber nicht. Denn laut Staatsanwaltschaft hatte er Patienten behandelt. Martin Wortmann

\title{
MVZ dürfen freie Arztsitze nicht bunkern
}

MVZ können frei gewordene Arztstellen nicht unbegrenzt freihalten. Für die Nachbesetzung haben sie jetzt in der Regel sechs Monate Zeit. Für Teilzeitstellen deutlich unter $50 \%$ sieht es zeitlich besser aus.

$\mathrm{m}$ entschiedenen Fall ging es um die

Nachbesetzung einer viertel Stelle im MVZ eines katholischen Krankenhauses im Bereich Nordrhein. Die Stelle wurde Ende Februar 2006 frei, erst im Juni 2007 beantragte das MVZ die Nachbesetzung. Der Berufungsausschuss hielt dies für $\mathrm{zu}$ spät und lehnte den Antrag ab.

Das Bundesozialgericht (BSG) nahm den Streit nun zum Anlass für eine grundsätzliche Klärung und grenzte die Nachbesetzung im MVZ von der in einer regulären Praxis ab. Zu Einzelpraxen hatte der Vertragsarztsenat entschieden, dass eine Nachbesetzung möglich ist, solange noch einsatzfähige Praxisräume vorhanden sind - solange also letztlich eine Weiterbehandlung des bisherigen Patientenstamms noch möglich ist. Diese Rechtsprechung ist jedoch auf ein MVZ nicht anwendbar, so das BSG. Denn sie würde bedeuten, dass ein MVZ Vertragsarztsitze faktisch unbegrenzt blockieren könnte. Dies wäre mit den Strukturprinzipien der Bedarfsplanung nicht vereinbar. Aus den gesetzlichen Gründungs- und Zulassungsvoraussetzungen eines MVZ leitete der BSG-Vertragsarztsenat nun eine Frist von sechs Monaten für die Nachbesetzung ab. Wird die Stelle bis dahin nicht neu besetzt, fällt sie weg (Aktenzeichen: B 6 KA 23/11 R).

Martin Wortmann
Abrechnungstipp!

\section{Bei dokumentierter Rötelnimpfung keine Serologie}

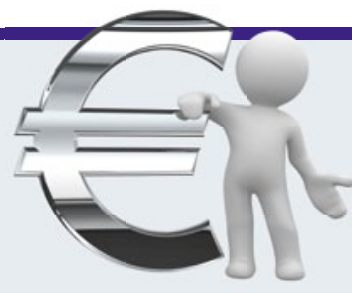

— Entsprechend den aktuellen STIKO-Empfehlungen gibt

es neue Regelungen im Rahmen der Mutterschafts- und der SchwangerschaftsabbruchRichtlinie. Die Änderung der Mutterschafts-Richtlinien trat am 19. August 2011 in Kraft:

_ Bestimmung der serologischen Rötelnimmunität nur noch bei Schwangeren, bei denen ein Nachweis über zwei erfolgte Rötelnimpfungen nicht vorliegt.

_Als weitere Änderung hat der G-BA in der Mutterschaftsrichtlinie beschlossen, gesunden Schwangeren eine Impfung gegen die saisonale Influenza im zweiten Trimenon zu empfehlen, bei erhöhter gesundheitlicher Gefährdung infolge eines Grundleidens auch schon im ersten Trimenon. Diese Impfung ist wie jede andere Impfung keine im EBM enthaltene Leistung. Sie wird vergütet aufgrund von den einzelnen KVen abgeschlossener Impfvereinbarung mit den jeweiligen Landesverbänden der Krankenkassen. Die einzelnen Ziffern sollten Sie bei Ihrer KV erfragen.

Die Änderung der Richtlinie zur Empfängnisregelung und zum Schwangerschaftsabbruch (ESA-RL), in Kraft getreten am 20.8.2011, wurde ebenfalls durch eine Ergänzung zur Rötelnimmunität ergänzt. „Bei Frauen mit dokumentierter zweimaliger Rötelnimpfung ist von Immunität auszugehen. Eine AK-Bestimmung ist hier nicht erforderlich. Frauen mit fehlender Immunität soll die Rötelnimpfung bzw. deren Komplettierung empfohlen werden." Fazit: Erst nach der Entscheidung des G-BA werden STIKO-Empfehlungen auch für die EBM-Abrechnung bzw. für die Abrechnung nach den Impfvereinbarungen tatsächlich relevant. Beim Immunstatus Röteln ist es jetzt so weit. 